\title{
RBMulticast: Receiver Based Multicast for Wireless Sensor Networks
}

\author{
Chen-Hsiang Feng, Wendi B. Heinzelman \\ Department of Electrical and Computer Engineering \\ University of Rochester \\ Rochester, NY, USA \\ Email:\{feng,wheinzel\}@ece.rochester.edu
}

\begin{abstract}
Multicast routing protocols typically rely on the apriori creation of a multicast tree (or mesh), which requires the individual nodes to maintain state information. In sensor networks where traffic is bursty, with long periods of silence between the bursts of data, this multicast state maintenance adds a large amount of overhead for no benefit to the application. Thus, we have developed a stateless receiver-based multicast protocol that simply uses a list of the multicast members (e.g., sinks), embedded in packet headers, to enable receivers to decide the best way to forward the multicast traffic. This protocol, called RBMulticast (Receiver-Based Multicast), exploits the knowledge of the geographic locations of the nodes to remove the need for costly state maintenance (e.g., tree/mesh/neighbor table maintenance), making it ideally suited for sensor network multicast applications. RBMulticast was implemented in TinyOS and tested using a sensor network implementation as well as TOSSIM simulations. Both simulation and experimental results confirm that RBMulticast provides high success rates without the burden of state maintenance.
\end{abstract}

\section{INTRODUCTION}

Communication in sensor networks is hindered by the limited energy capacity of the individual sensor nodes. Consequently, reducing the total number of packets transmitted throughout the network is essential for power conservation. For sensor networks with multiple sink nodes, multicast routing is an ideal approach to manage and reduce network traffic. Reducing the number of packets transmitted when multicasting data requires both shorter routing paths from the multicast source to the multicast members, as well as improved efficiency in terms of the total number of links the packets traverse to get to all the multicast members, i.e., the packet should be split off to different routing branches only when necessary. Shorter routing paths lead to reduced packet delay, and improved efficiency leads to a reduction in the energy consumption from transmitting fewer packets. These two properties are usually contradictory to each other, and algorithms must make a trade-off to best fit their requirements.

In this paper, we develop a novel multicast protocol called RBMulticast (Receiver-Based Multicast). RBMulticast is a completely stateless multicast protocol, using only location information with no tree creation or maintenance or even neighbor table maintenance, which makes it ideally suited for sensor networks. Packet routing and splitting packets into multiple routes relies solely on the location information of each multicast member, which is assumed to be known.
RBMulticast is a receiver-based protocol (as with the ExOR protocol [1]), which means that a sender can transmit packets without specifying the next hop node, because the potential receivers of this packet make the decision of whether or not to forward this packet in a distributed manner. This approach for transmitting packets means that routing is a result of the joint decisions of all participating nodes. Therefore, no routing tables are required within the sender node, as potential receivers decide on a valid route.

RBMulticast was motivated by the cross-layer protocol XLM [2], which is a receiver-based unicast protocol designed for WSNs. As in XLM, RBMulticast assumes a MAC protocol whereby receivers contend for channel access based on their assessed contribution towards forwarding the packet. Nodes with more energy and better links and nodes that make the most forward progress to the destination will contend earlier and hence have a higher chance to become the next-hop node. In RBMulticast, we extend this idea for multicast routing by using the concepts of a "virtual node" and a "multicast region" for forwarding packets closer to the destination multicast members and determining when packets should be split into separate routes to finally reach the multicast members.

We implemented RBMulticast in TinyOS and performed experiments using a Tmote Sky test-bed as well as TOSSIM simulations. Results of these experiments show that RBMulticast maintain high success rate (e.g., over $80 \%$ ) in highly dynamic networks, where nodes only receive packets in a 10 ms interval and change radio state every $100 \mathrm{~ms}$. This level of performance in such dynamic networks is not easy using other multicast approaches because nodes must keep updated information about the network. We believe that RBMulticast is lightweight and robust, making it ideally suited for multicast applications in dynamic sensor networks.

\section{RELATED WORK}

Existing multicast protocols for WSNs and mobile ad hoc networks (MANETs) generally use a tree to connect the multicast members. For example, the Takahashi-Matsuyama heuristic can be used to incrementally build a Steiner tree for multicast routing [3]. Additionally, multicast algorithms rely on routing tables maintained at intermediate nodes for building and maintaining the multicast tree [4]. 
Due to the specificities of WSNs, knowing sensor nodes' locations is a reasonable assumption. In the location-based approach to multicast routing, nodes obtain location information by default as an application requirement (e.g., a home fire alarm would know where it is located) or as provided by a system module (e.g., GPS or a location-finding service). If location information is known, multicast routing is possible based solely on location information without building any external tree structure. For example, PBM [5] weights the number of next hop neighbor nodes and total geographic distance from the current node to all destination nodes and compares this to a predefined threshold to decide whether or not the packet should be split. Geocast [6] delivers multicast packets by restricted flooding. Nodes forward multicast packets only if they are in the Forwarding Zone calculated at run time from global knowledge of location information.

RBMulticast differs from these approaches in that it is completely stateless and hence no costly state maintenance is required. PBM [5] uses a similar idea of stateless multicast but requires information about neighbor nodes. RBMulticast further eliminates the requirement of knowing a node's neighbors by using a receiver-based mechanism, and only the location of the nodes is needed for multicast packet routing. Additionally, RBMulticast includes a list of the multicast members in the packet header, which prevents the overhead of building and maintaining a multicast tree at intermediate sensor nodes, because all the necessary information for routing the packet is included within the packet header. We believe that RBMulticast requires the least state of any multicast routing protocol and is thus ideally suited for WSNs

Receiver-based communication is a different way of thinking about protocol design in that decisions are not required to be made at the sender side but instead are made at the receiver side. For example, a source node in ExOR [1] broadcasts packets that include a potential forwarders' list inside the header, and these potential forwarders will contend to forward the packet through the use of different back-off times, which depend on the network distance to the destination. A source node in XLM [2] broadcasts packets with the destination's geographic location in the header, and every receiver contends to forward the packet through the use of different back-off times, which depend on the geographic distance to the destination. In other words, in receiver-based routing, decision-making is deferred to the possible receivers, who make decisions in a distributed manner.

Receiver-based routing is different from "On-demand" or "Reactive" routing in that reactive routing calculates a route at the time a packet is sent down to the MAC layer. For example, AODV [7] begins transmission by first sending a "RouteRequest" to create temporary routes among intermediate nodes and then transmits data packets through this route. The ability to transmit data without requiring a route to be formed is enabled via extra knowledge in the MAC layer and join decisions of sensor nodes. For example, nodes could be assigned an ID in a structured manner and hence next hop nodes are implied in the destination address itself. In this case, packets are broadcast by the MAC layer, and only potential next-hop nodes relay it to the destination. As another example, nodes may have statistics (e.g., energy, channel quality) that could assist in making forwarding decisions. A source node can send an RTS packet, enabling potential receivers to contend for the ability to forward the packet, with the receiver node that has the best route being the first to return a CTS to receive this packet.

\section{RBMulticast PRotocol Description}

RBMulticast is a receiver-based Network layer protocol that performs multicast routing based on multicast members' location information. There are some assumptions for RBMulticast. First, we assume that there exists a Location Service module inside the protocol stack, which accepts a query of the network address of a node and returns the 2-dimensional coordinates of that node. Second, we assume a receiver-based MAC protocol exists in the Link layer. The next hop of a route should be decided among potential receivers (e.g., through receiver contention). Third, we assume that the receiver-based Link layer only needs the sender node's location and the destination node's location to decide the next hop route, and that both are provided in the MAC packet.

We also assume that the "void" (hole) problem in geographic routing is solved implicitly in the MAC layer. Perimeter stateless routing, as used in GPSR [8], is a possible solution to holes in the network, but this requires a neighbor table to generate a graph representation of the network, which is against our ultimate goal of a completely stateless protocol. Instead, in our implementation, we combine the receiver-based MAC protocol with the "Water Flowing" idea from TORA [9], where each node has an implicit "water height" and packets cannot flow from a lower to a higher water level. Nodes increase their height when they determine that they are near a void area, which will automatically prevent packets from using this route in the future.

\section{A. RBMulticast Overview}

Nodes in RBMulticast create what we call "multicast regions" centered around themselves. There are several ways to create these regions (see Section III-B), but for simplicity it can be assumed that each multicast region corresponds to one quadrant of the network, for a grid centered at the node, as shown in Figure 1. When a user initiates a request to send a packet to a multicast group, data are passed down to the RBMulticast module in the Network layer of the protocol stack. Once the RBMulticast module gets this packet, it retrieves the group list from its group table, compares the group nodes' location to the multicast regions, and calculates a virtual node location for each multicast region. RBMulticast replicates the packet for each multicast region that contains one or more multicast members and appends a header consisting of a list of destination nodes (multicast members) in that region, TTL (Time to Live) value, and a checksum value. The destination of the packet is a "virtual node" for that multicast region, which can be determined in several ways (see Section III-C), 
but for simplicity it can be assumed to be the geometric mean of the locations of all the multicast members in the multicast region. In the end, all packets for all multicast regions are passed down to the MAC layer, which broadcasts them to the node's neighbors. The node closest to the location of the virtual node (as determined by receiver-based contention at the MAC layer) will take responsibility for forwarding the packet. The procedures for transmitting packets are summarized in pseudo code in Algorithm 1.

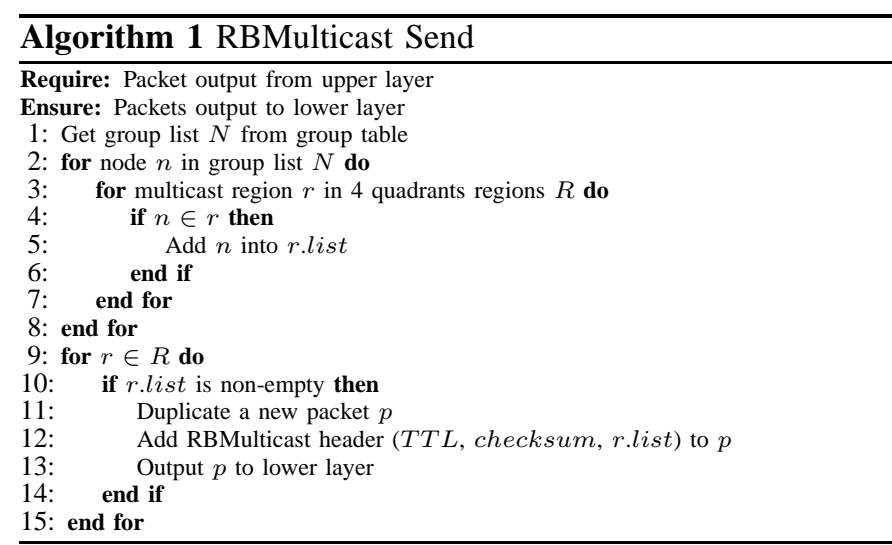

When a node receives a multicast packet, the packet is passed up from the Link layer to the RBMulticast protocol. RBMulticast first examines the checksum in the packet header, and drops the packet if any corruption exists in the packet. It then retrieves the destination nodes list from the RBMulticast packet header. If this node is inside the destination list, it removes itself from the list and passes a copy of the packet up to the upper layers in the protocol stack. RBMulticast then checks the TTL value and drops the packet if the TTL is lower than a threshold. Finally, if there still remain nodes in the destination list, multicast regions and virtual nodes will be recalculated, and new packets will be generated if required. The packets (one per multicast region that contains multicast members) are then passed down to the Link layer for transmission. The procedures for receiving packets are summarized in pseudo code in Algorithm 2.

Figure 1 gives an example of how RBMulticast is employed in complex WSNs. The first two multicast regions (in the south-west and north-west quadrants) contain only one multicast member each, and thus a packet is sent directly to these multicast destinations. The third multicast region has three multicast members, and thus a single packet is sent to a virtual node (with label 3 in the figure), which is located at the geometric mean of the locations of the multicast members. The fourth multicast region has no multicast members, and hence no packet is transmitted into this region. Once the packet sent towards virtual node 3 reaches an intermediate node for which the multicast members are no longer in the same multicast region, the node will split off packets to each of the multicast regions, using either virtual nodes if there are two or more multicast members in the multicast region or sending the packet directly to the multicast member if it is the
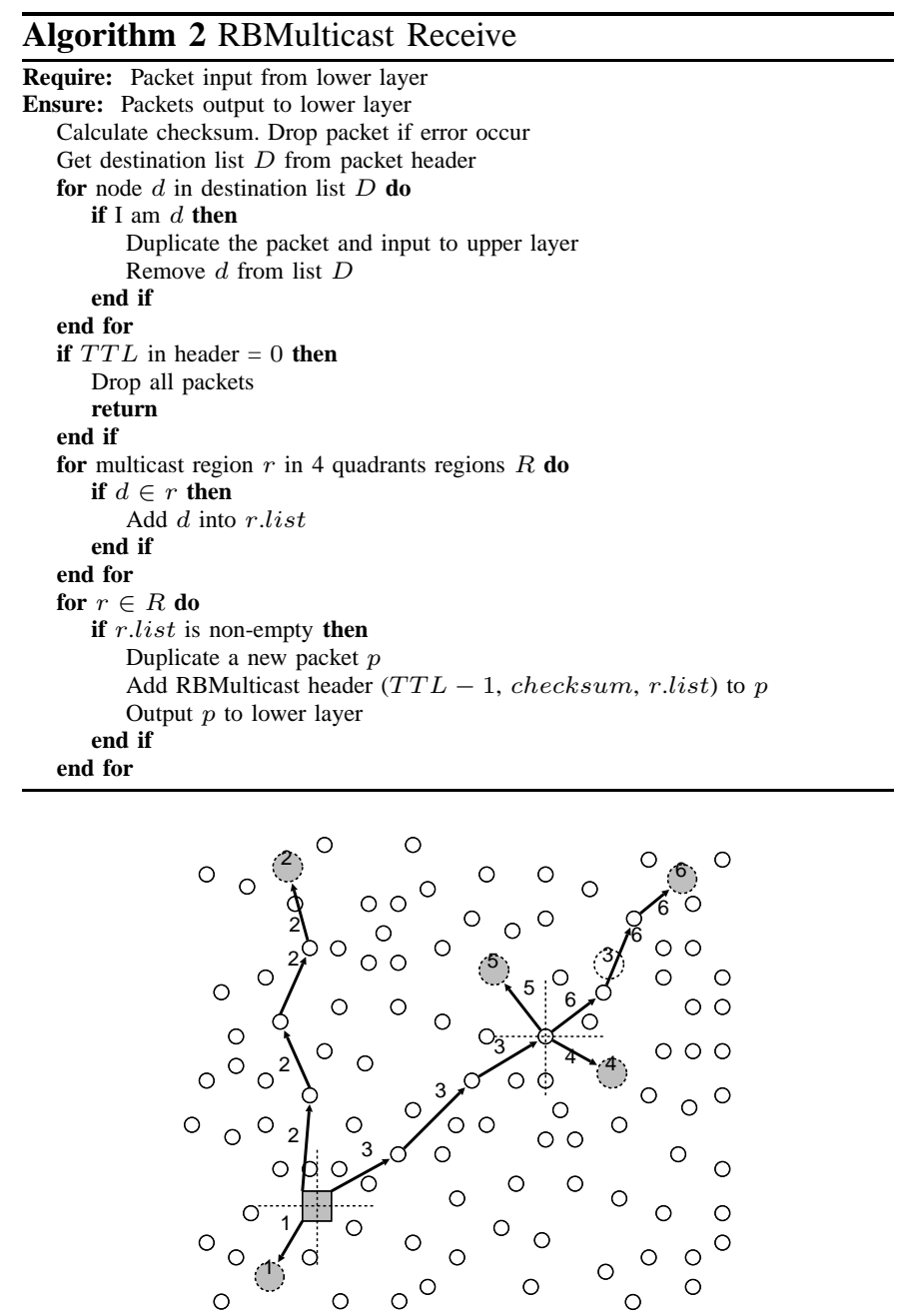

Fig. 1. Example showing how RBMulticast delivers multicast packets. The source node is the square node. Multicast members are shaded circles, and virtual nodes are dotted circles. Because every destination node will become a virtual node at the end, they are all shown with dotted circles. The number on the side of the lines indicate the destination of that packet.

only one in the multicast region.

\section{B. Multicast Regions}

Once a node receives a multicast packet (from the application or from a previous hop node), it divides the network into multicast regions, and it will split off a copy of the packet to each region that contains one or more multicast members. We show two possible divisions of the network into multicast regions in Figure 2(a) and 2(b).

Dividing space into three 120 degree pieces is a straightforward approach because it resembles a Steiner tree in that every node has three branches. To separate space into 120 degree regions, we must calculate the angle to each destination node. Calculating this angle relies on trigonometric calculations and hence requires floating point operations. Most CPUs in current sensor nodes do not support floating point operation due to the demand for low power and low cost, and hence floating point operations must be simulated by integer operations and are 


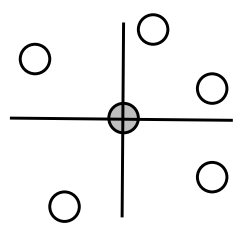

(a)

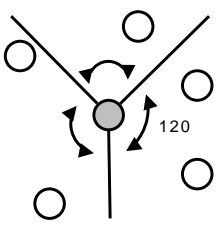

(b)

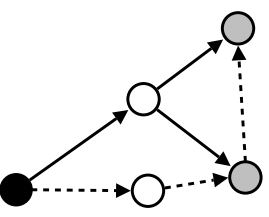

(c)
Fig. 2. a) and b) are two possible ways to divide a space into multicast regions: a) dividing the space into four quadrants, and b) dividing the space into three 120 degree pieces. c) demonstrates how to choose a next hop node. The solid node is the source node, and the gray nodes are the multicast members. The solid line is the route when choosing a target node near the geographic mean of the multicast members, and the dotted line is the route when choosing a target node close to the nearest multicast member. We can see that the longest distance is two hops distance in the first case, and it is three hops distance in the second case.

thus expansive. Moreover, multicast regions must recalculated in each hop of packet delivery. We believe that this will becomes an excessive burden on sensor nodes and is therefore unacceptable.

The quadrants approach in some cases sends more packets than the 120 degree angle approach, but the multicast region calculation only needs two comparisons ( $\mathrm{X}$ and $\mathrm{Y}$ axes) for each multicast member and is extremely fast. We believe that it is thus preferable and apply this 4 quadrants approach in our implementation of the RBMulticast protocol.

\section{Virtual Node}

Network layer multicast protocols, which require multiple destinations, are built on top of Link layer protocols that typically allow only a single (unicast) or all (broadcast) destinations. Possible ways to adapt the need for multiple multicast destinations to a MAC layer that can only handle a single destination are choosing a node near the geographic mean of the multicast members, or choosing a node near the nearest multicast node, as shown in Figure 2(c). The geographic mean approach has fewer hops in general.

In RBMulticast, because we assume no knowledge of neighbor nodes and routing tables, we assign a "virtual node" located at the geographic mean of the multicast members for each multicast region. This virtual node is used as an imaginary destination for the multicast packet in that region. The virtual nodes (as shown in Figure 1) are not necessarily reachable or even physically exist. The idea behind this is that even if a virtual node does not exist, we can still find a next hop route using the assumed receiver-based MAC protocol to get the packet closer to the location of the virtual node.

On the other hand, when using the nearest multicast node as the destination, all node addresses physically exist and virtual nodes are not necessary. However, with this approach we lose the advantage of shorter routes as shown in Figure 2(c).

\section{Destination List}

The goal of our approach is to keep intermediate sensor nodes from having to store any multicast routing state. This is possible only if all required information to multicast a packet

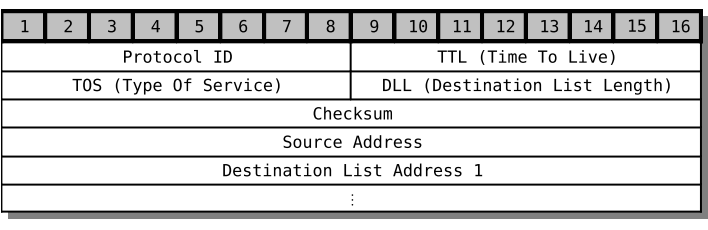

Fig. 3. Packet header of the RBMulticast protocol.

is carried along with the packet. The question is how much information the multicast packet needs to carry for successful delivery to all multicast members.

Because we assume a receiver-based MAC layer, the next hop is determined by a joint decision among potential receivers. The RBMulticast header does not need to carry any state for routing the packet.

However, we still need to decide when the packet must be split off to different destinations. This is usually implied by tree branches in tree-based multicast approaches. Because of the location information assumption, we use multicast regions to decide when packets must be split off without any tree structure. A packet will be split off to each multicast region if multicast members exist in that region. Therefore, a destination list is the only requirement for multicast packet delivery and must be carried inside the packet header.

\section{E. RBMulticast Header}

Figure 3 offers an example of an RBMulticast header. The first byte Protocol ID is for packet switching in the protocol stack [10]. TTL (Time To Live) provides a maximum time, in hop number, that a packet should last in the network. TOS (Type Of Service) indicates 4 kinds of packets in RBMulticast, which are "data", "join", "leave", and "update" packets. The update packets are used in group management and periodic group list update. DLL (Destination List Length) indicates how many nodes are in the node list, and thus will determine the length of the header. The RBMulticast header size is not fixed because the destination list length is variable. Source Address stores the RBMulticast group address of this packet and Destination List Address stores the addresses of the DLL destination nodes.

The maximum number of multicast members allowed in a group is restricted by the packet size. For packets in the 802.15 .4 standard, maximum packet size is 128 bytes, and hence the maximum number of nodes in the destination list is around 50, if the data payload is not considered. However, it is unlimited if the MAC layer can support segmentation or aggregation of oversize packets.

One point worth noting is the overhead introduced by the destination list. As with any multicast protocol that uses a destination list, the packet header length will increase linearly with the number of destination nodes, and thus RBMulticast is not designed for applications with an extremely large number of multicast members. The energy for sending the extra bytes of data is negligible, but the probability of packet collision will increase, which introduces extra energy consumption. The impact of packet length on energy consumption can be reduced 


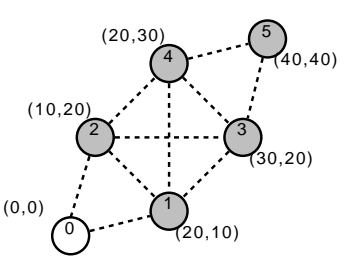

(a)

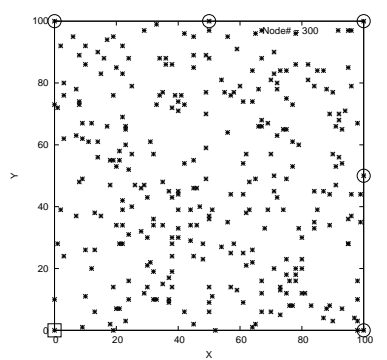

(b)
Fig. 4. a) The experimental network for comparing results in the Tmote Sky implementation and the TOSSIM simulations. Node 0 is the source node and the shaded nodes are multicast destination nodes. b) Large scale experimental network used for simulations. The source node is indicated by a square in the left corner, and the multicast receiver nodes are indicated by circles located at the boundary of the region.

by adjusting the power control of the MAC protocol, as shown in [11].

\section{F. Group Management}

Every node owns a multicast group and acts as a group head. Nodes join and leave a group by sending "join" and "leave" packets to the group head. Join and leave packets are multicast packets with destination lists that contain only the group head address.

\section{EXPERIMENTAL RESULTS}

We evaluate the performance of RBMulticast through a test-bed implementation using Tmote Sky motes and through simulation using TOSSIM. RBMulticast is implemented in a new sensor network protocol stack framework called UPS [10], with a MAC layer protocol XLM/MAC installed; this MAC protocol is based on the MAC functionality of the XLM [2] cross-layer protocol. XLM/MAC is a receiver based protocol that uses location information in RTS/CTS/DATA/ACK handshakes, and nodes jointly decide the next hop node through receiver contention. Receivers close to the destination and with low contention and high remaining energy have a higher priority to become the next hop node and to route packets.

We test the RBMulticast protocol in a highly dynamic scenario, where nodes have a very short duty cycle time of $100 \mathrm{~ms}$. For example, a duty cycle of 0.2 (20\%) means that in every $100 \mathrm{~ms}$, nodes will turn their radios on for $20 \mathrm{~ms}$ and then go to sleep for the remaining $80 \mathrm{~ms}$ if not transmitting/receiving. We use this highly dynamic scenario to demonstrate the advantages of stateless multicast. That is, RBMulticast can achieve high success rates and low latency in a highly dynamic scenario where structured (e.g., tree) approaches are difficult to employ.

For the first experiment, we collect data from six Tmote Sky sensor nodes arranged in the topology shown in Figure 4(a). The statistics shown in Figure 5 compare the simulation results of TOSSIM simulations with the results of the Tmote Sky experiments. In this figure, we see that the implementation results match closely with the simulation results. The success

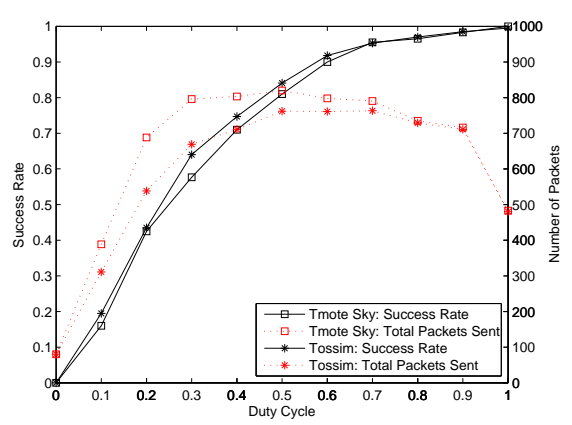

Fig. 5. Comparison of Tmote Sky implementation to TOSSIM simulations.

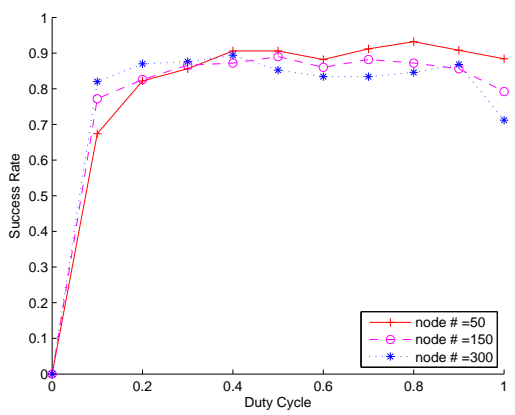

Fig. 6. Average success rate for the large-scale network.

rate is slightly higher in the TOSSIM simulations than in the Tmote Sky implementation. This is because the Tmote Sky mote (which contains a CC2420 radio chip) requires $8 \mathrm{~ms}$ plus some CPU overhead to send/receive a packet, and this is on the same order of the time it takes the radio to change its status in our experiment (e.g., $20 \mathrm{~ms}$ for a duty cycle of 0.2 ). In this experiment we set the MAC layer re-try limit to 4 times before a packet is dropped, and the increased number of packets sent for the Tmote Sky implementation is hence due to the extra resent RTS packets. From these results, we conclude that the TOSSIM simulations closely track the real implementation results.

In the next experiment, we simulated the use of RBMulticast under different network densities for large-scale WSNs, as shown in Figure 4(b). There are a total of either 50, 150 or 300 nodes randomly distributed throughout the simulation area. The source node is located at the bottom left corner $(0,0)$, and the multicast receiver nodes are scattered over the boundary of the region. In order to increase the success rate in the low duty cycle cases, we modify the MAC protocol to try to send the RTS packet up to 30 times before the packet is dropped.

We show the TOSSIM simulation results for the success rates at different duty cycles and for different node densities in Figure 6. The success rates for all three nodes densities are around 0.8 and 0.9 , with a slight decrease in the low duty cycle regime. We believe that the reason RBMulticast does not achieve $100 \%$ success rate is due to a MAC layer limitation. Specifically, when an intermediate node splits packets to send 


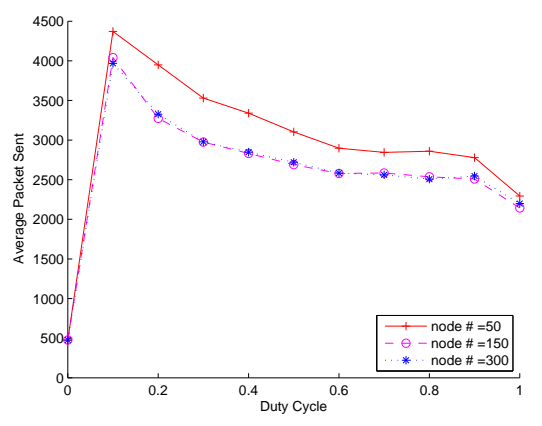

Fig. 7. Total number of packets sent, including all MAC layer packets (e.g., RTS/CTS/DATA/ACK).

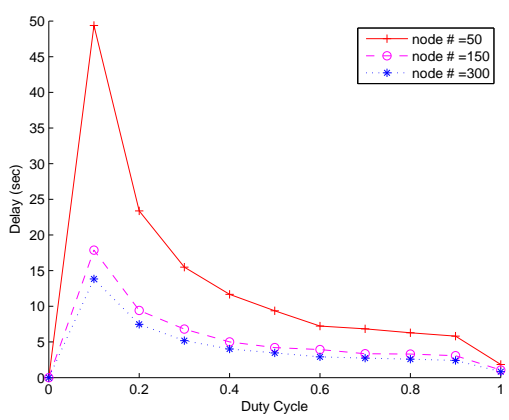

Fig. 8. Average packet delay for the large-scale network.

to the different "multicast regions," it injects multiple packets into the network in a short period of time, thus causing local congestion in that area. This can be improved by using more advanced MAC layer protocols with congestion control capabilities.

Figure 7 shows the total number of packets sent in the simulations. Because the 50 node network is fairly sparse, more repeated RTS packets are required to establish connections between nodes, especially at low duty cycles since the sleep times are not synchronized. Because the node density and redundancy is high in the 150 and 300 node cases, fewer re-sent RTS packets are required and hence this results in a lower total number of packets sent. We expect that the number of packets sent will remain the same as the number of nodes increases further. We must emphasize that all packets in RBMulticast are purely due to data transmission, no extra control packets are needed, and thus the total number of packets sent does not increase as the number of nodes increases.

Figure 8 shows the average packet delay for all multicast nodes. In the low node density case (e.g., 50 nodes), packet delay is large, as expected because the high RTS packets loss rate results in large back-off times to resend RTS packets. On the other hand, for the dense networks with 150 and 300 nodes, the packet delay is reduced and approaches an optimal value of around 1 second.

\section{CONClusion}

Current multicast protocols generally rely on various tree structures and hence intermediate nodes need to maintain tree states or routing states for packet delivery. In this paper, we presented a new stateless multicast protocol for WSNs called Receiver-Based Multicast (RBMulticast). RBMulticast uses geographic location information to route multicast packets, where nodes divide the network into geographic "multicast regions" and split off packets depending on the locations of the multicast members. RBMulticast stores a destination list inside the packet header; this destination list provides information on all multicast members to which this packet is targeted. Thus, there is no need for a multicast tree and therefore no tree state is stored at the intermediate nodes. RBMulticast also utilizes a receiver-based MAC layer to further reduce the complexity of routing packets. Because we assume that the receiver-based MAC protocol can determine the next hop node in a distributed manner, the sender node does not need a routing table or a neighbor table to send packets but instead uses a "Virtual Node" as the packet destination. Thus RBMulticast requires the least amount of state of any existing multicast protocol. Our simulations and implementation of RBMulticast showed that with the Receiver-based MAC protocol XLM/MAC, RBMulticast can achieve high success rates and low latency, making RBMulticast well suited for dynamic sensor network environments.

\section{REFERENCES}

[1] S. Biswas and R. Morris, "Opportunistic routing in multi-hop wireless networks," SIGCOMM Computer Communications Review, vol. 34, no. 1, pp. 69-74, 2004.

[2] I. Akyildiz, M. Vuran, and O. Akan, "A cross-layer protocol for wireless sensor networks," in Proc. of CISS 2006, March 2006.

[3] K. Chen and K. Nahrstedt, "Effective location-guided tree construction algorithms for small group multicast in manet," INFOCOM. Twenty-First Annual Joint Conference of the IEEE Computer and Communications Societies. Proceedings. IEEE, vol. 3, pp. 1180-1189, 2002.

[4] A. Okura, T. Ihara, and A. Miura, "Bam: branch aggregation multicast for wireless sensor networks," Mobile Adhoc and Sensor Systems Conference, 2005. IEEE International Conference on, pp. 10 pp.-, Nov. 2005.

[5] M. M. andand Holger Fuler, J. Widmer, and T. Lang, "Positionbased multicast routing for mobile ad-hoc networks," SIGMOBILE Mob. Comput. Commun. Rev., vol. 7, no. 3, pp. 53-55, 2003.

[6] Y.-B. Ko and N. H. Vaidya, "Geocasting in mobile ad hoc networks: Location-based multicast algorithms," wmcsa, vol. 0, p. 101, 1999.

[7] "Ad-hoc on-demand distance vector routing," Mobile Computing Systems and Applications, 1999. Proceedings. WMCSA. Second IEEE Workshop on, pp. 90-100, 1999.

[8] B. Karp and H. T. Kung, "GPSR: greedy perimeter stateless routing for wireless networks," in MobiCom '00: Proceedings of the 6th annual international conference on Mobile computing and networking. New York, NY, USA: ACM, 2000, pp. 243-254.

[9] V. D. Park and M. S. Corson, "A highly adaptive distributed routing algorithm for mobile wireless networks," infocom, vol. 00, p. 1405, 1997.

[10] "UPS: Unified Protocol Stack for Wireless Sensor Networks," under submission.

[11] H. Chen and Y. Li, "Performance model of ieee 802.11 dcf with variable packet length," Communications Letters, IEEE, vol. 8, no. 3, pp. 186188, March 2004. 